\title{
Millimeter Wave Cloud Radar Handbook
}

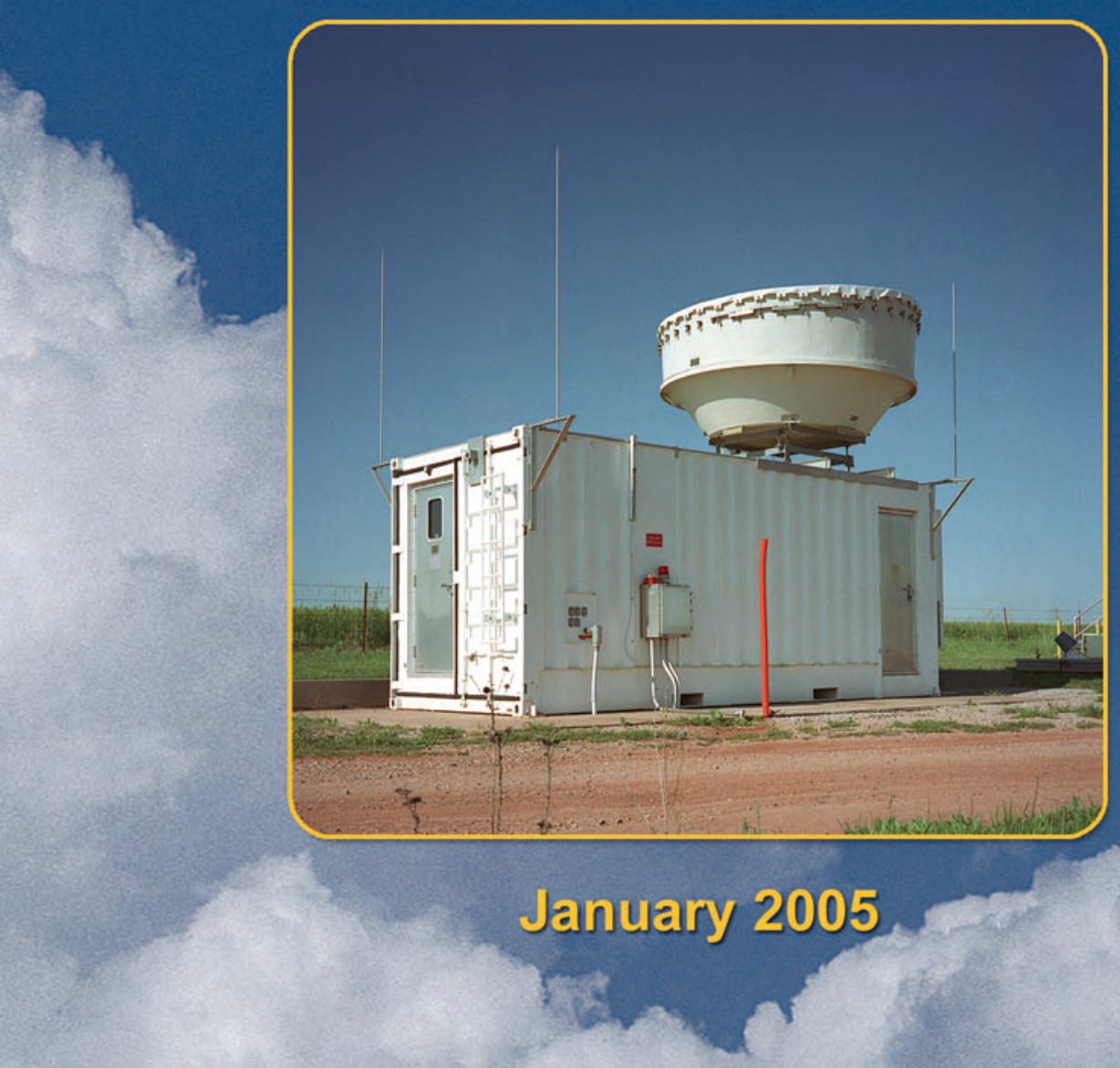

Work supported by the U.S. Department of Energy Office of Science, Office of Biological and Environmental Research 
ARM TR-018

\title{
Millimeter Wave Cloud Radar (MMCR) Handbook
}

January 2005

\author{
K. B. Widener \\ K. Johnson
}

Work supported by the U.S. Department of Energy, Office of Science, Office of Biological and Environmental Research 


\section{Contents}

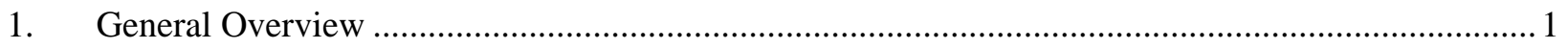

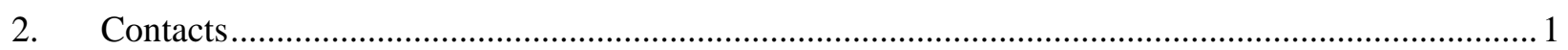

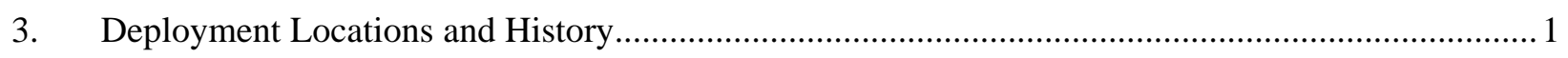

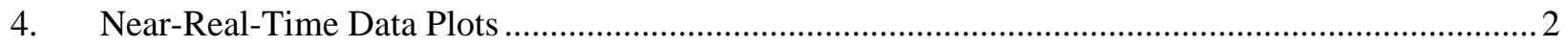

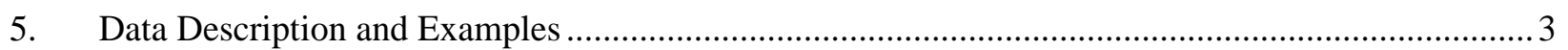

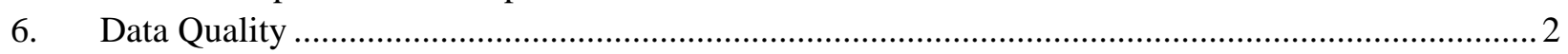

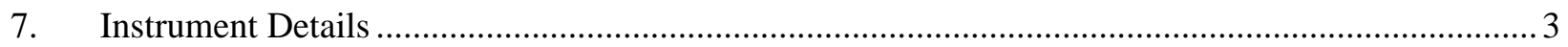

Figures

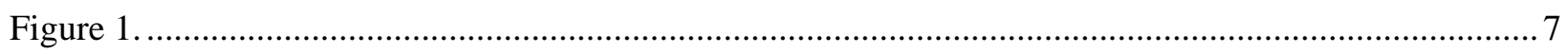

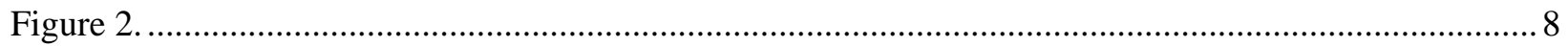

\section{Tables}

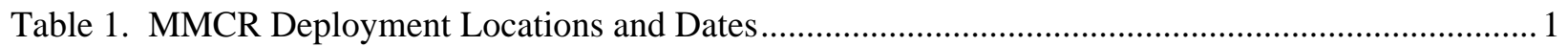

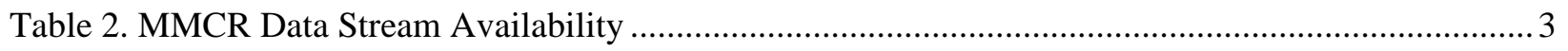

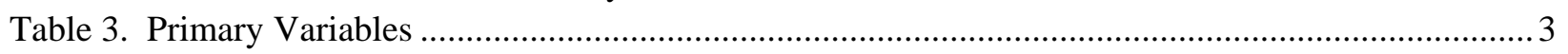

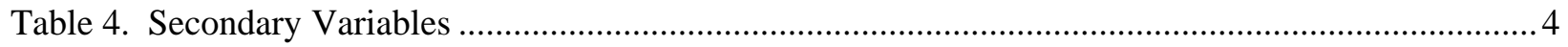

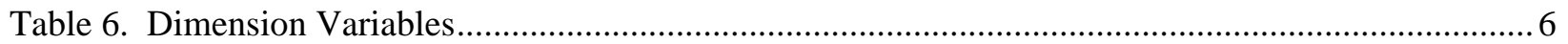

Table 7. ARM SGP Site Operation Parameters 1997.09.15 - 2004.08.10 ….......................................... 5

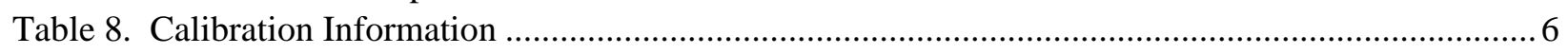

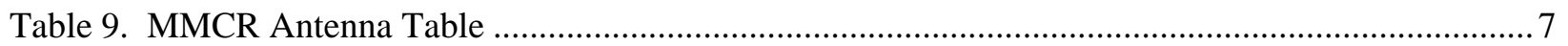




\section{General Overview}

The millimeter cloud radar (MMCR) systems probe the extent and composition of clouds at millimeter wavelengths. The MMCR is a zenith-pointing radar that operates at a frequency of $35 \mathrm{GHz}$. The main purpose of this radar is to determine cloud boundaries (e.g., cloud bottoms and tops). This radar will also report radar reflectivity (dBZ) of the atmosphere up to $20 \mathrm{~km}$. The radar possesses a doppler capability that will allow the measurement of cloud constituent vertical velocities.

\section{Contacts}

\subsection{Mentor}

Kevin Widener

Pacific Northwest National Laboratory

P.O. Box 999

Richland, WA 99352

Phone: 509-375-2487

Fax: 509-375-6736

kevin.widener@pnl.gov

Karen Johnson

Environmental Sciences Department

Brookhaven National Laboratory

Upton, NY 11973

Phone: 631-344-5952

Fax: 631-344-2060

kjohnson@bnl.gov

\subsection{Instrument Developer}

Ken Moran, Project Leader

NOAA Environmental Research Laboratories

Environmental Technology Laboratory

325 Broadway, R/E/ET4

Boulder, CO 80303

Phone: 303- 497-6575

Ken.Moran@noaa.gov

\section{Deployment Locations and History}

Table 1. MMCR Deployment Locations and Dates

\begin{tabular}{|c|c|l|c|}
\hline Location & Date Installed & Date Removed & Status \\
\hline SGP/C1 & $1996 / 11 / 08$ & & Operational \\
\hline NSA/C1 & $1998 / 03 / 25$ & & Operational \\
\hline TWP/C1 & $1999 / 06 / 14$ & & Operational \\
\hline
\end{tabular}


Table 1. (cont'd)

\begin{tabular}{|c|c|c|c|}
\hline Location & Date Installed & Date Removed & Status \\
\hline TWP/C2 & $1998 / 10 / 22$ & & Operational \\
\hline TWP/C3 & $2002 / 03 / 06$ & & Operational \\
\hline
\end{tabular}

The first five MMCRs were built by the National Oceanic and Atmospheric Administration's (NOAA's) Environment Technology Laboratory (ETL) in Boulder, Colorado. The first unit is now operating at the Southern Great Plains (SGP) Central Facility. The second unit is currently operating at the North Slope of Alaska/Adjacent Artic Ocean (NSA/AAO) site, and the third and forth units are located at the Tropical Western Pacific's Atmospheric Radiation and Cloud Station (TWP's ARCS-1) on Manus Island and ARCS-2 on Nauru Island. The fifth unit is a hot spare/development radar located at NOAA/ETL. The sixth radar was built by Radian International; it is currently installed at Darwin, Northern Territory, Australia at the ARCS-3 site.

In addition to the ARM MMCRs, NOAA/ETL fielded an identical radar during the Surface Heat Budget of the Arctic Ocean (SHEBA) (http://sheba.apl.washington.edu) experiment from the fall of 1997 to the fall of 1998.

A new MMCR data processor, the C-40, was developed by NOAA/ETL and installed at the SGP and NSA sites in September 2003 and April 2004, respectively. Along with the new MMCR radar computer, the C-40 provides faster data collection and much improved instrument reliability. Upgrades are planned for the TWP sites in the coming year.

\section{Near-Real-Time Data Plots}

Near-real-time MMCR data plots are available from the Data Quality Office (DQO) for each site. Thumbnail images of the most recent data are available from the ARM DQHandS Plot Browser website. Select the site of interest and select the associated MMCR data stream. For NSA, choose 'nsammcrmom,' for SGP select 'sgpmmcrmom,' and for the TWP sites, select 'twpmmcrcal.' Then choose either the 'list' or 'thumbnail' search option. The 'list' option provides a list of available plots; the 'thumbnail' option presents thumbnails images. The most recent week of data is the default date range.

DQO plots can also be accessed individually via http://dq.arm.gov/PLOTS. MMCR plots for each site are accessible as listed below. In each case, select the date of interest from the page that appears:

NSA: http://dq.arm.gov/PLOTS/NSA/nsammcrmom/

SGP: http://dq.arm.gov/PLOTS/SGP/sgpmmcrmom/

TWP: http://dq.arm.gov/PLOTS/TWP/twpmmcrcal/

(Individual TWP site plots for C1, C2 are accessible once a date is selected. Plots for TWP-C3 are not yet available from the DQO.) 


\section{Data Description and Examples}

MMCR data are available from the ARM Data Archive in the following data streams: mmcrmom, mmcrcal, mmcrmoments, and mmcrspecmom. This table indicates which data streams are available at each site and time period:

Table 2. MMCR Data Stream Availability

\begin{tabular}{|l|l|c|}
\hline \multirow{2}{*}{ Site } & \multicolumn{2}{c|}{ Date Range } \\
\cline { 2 - 3 } & mmcrcal, mmcrmoments & \multicolumn{1}{c|}{ mmcrmom } \\
\hline NSA & 1999.03 - 2004.04.12 & 2004.04 .13 - Present \\
\hline SGP & 1996.11 - 2003.09.08 & 2003.09 .09 - Present \\
\hline TWP-C1 & 1999.08 - Present & \\
\hline TWP-C2 & 1998.11 - Present & \\
\hline TWP-C3 & 2002.03 - Present & \\
\hline
\end{tabular}

The data streams 'mmcrcal' and 'mmcrmoments' together make up a complete set of MMCR data. The ‘mmcrmom' data stream alone contains all the MMCR data fields.

\subsection{Data File Contents}

\subsubsection{Primary Variables and Expected Uncertainty}

The following tables show the primary quantities measured by the MMCR for the mmcrmom data stream, followed by the mmcrcal, mmcrmoments data streams.

Table 3. Primary Variables

mmcrmom Data Stream (current SGP, NSA)

\begin{tabular}{|l|l|l|}
\hline \multicolumn{1}{|c|}{ Variable } & \multicolumn{1}{|c|}{ Description } & \multicolumn{1}{c|}{ Uncertainty } \\
\hline Reflectivity & MMCR Reflectivity (time, height), in dBZ & $0.5 \mathrm{~dB}$ \\
\hline MeanDopplerVelocity & MMCR Mean Doppler Velocity (time, height), in $\mathrm{m} / \mathrm{s}$ & $0.1 \mathrm{~m} / \mathrm{s}$ \\
\hline SpectralWidth & MMCR Spectral Width (time, height), in m/s & $0.1 \mathrm{~m} / \mathrm{s}$ \\
\hline CircularDepolarizationRatio & MMCR Circular Depolarization Ratio (time, height), dB & \\
\hline
\end{tabular}

mmcrcal / mmcrmoments Data Streams (current TWP, historical SGP, NSA)

\begin{tabular}{|l|l|l|l|}
\hline \multicolumn{1}{|c|}{ Variable } & Data Stream & \multicolumn{1}{c|}{ Description } & Uncertainty \\
\hline Reflectivity & mmcrcal & MMCR Reflectivity (time-height), in dBZ & $0.5 \mathrm{~dB}$ \\
\hline MeanDopplerVelocity & mmcrmoments & $\begin{array}{l}\text { MMCR Mean Doppler Velocity (time-height), in } \\
\mathrm{m} / \mathrm{s}\end{array}$ & $0.1 \mathrm{~m} / \mathrm{s}$ \\
\hline SpectralWidth & mmcrmoments & MMCR Spectral Width (time, height), in m/s & $0.1 \mathrm{~m} / \mathrm{s}$ \\
\hline
\end{tabular}


The overall uncertainties for the primary quantities measured are as follows:

- Measurement accuracy: $0.5 \mathrm{~dB}$ over receiver dynamic range

- Doppler resolution: less than 0.1 meters/second.

\subsubsection{Definition of Uncertainty}

We define uncertainty as the range of probable maximum deviation of a measured value from the true value within a $95 \%$ confidence interval. Given a bias (mean) error $B$ and uncorrelated random errors characterized by a variance $\sigma^{2}$, the root-mean-square error (RMSE) is defined as the vector sum of these,

$$
R M S E=\left(B^{2}+\sigma^{2}\right)^{1 / 2} \text {. }
$$

( $B$ may be generalized to be the sum of the various contributors to the bias and $\sigma^{2}$ the sum of the variances of the contributors to the random errors). To determine the $95 \%$ confidence interval we use the Student's $t$ distribution: $t_{\mathrm{n} ; 0.025} \approx 2$, assuming the RMSE was computed for a reasonably large ensemble. Then the uncertainty is calculated as twice the RMSE.

\subsubsection{Secondary/Underlying Variables}

Table 4. Secondary Variables

mmcrmom Data Stream

\begin{tabular}{|l|l|}
\hline \multicolumn{1}{|c|}{ Variable } & \multicolumn{1}{c|}{ Description } \\
\hline GateSpacing & Gate Spacing (ns) for each mode \\
\hline InterPulsePeriod & Inter-Pulse Time Period (ns) for each mode \\
\hline MinimumDetectableReflectivity & $\begin{array}{l}\text { Minimum Detectable Reflectivity (dBZ), hourly for each mode \& } \\
\text { height }\end{array}$ \\
\hline ModeNum & Operating Set for each Record \\
\hline NoiseLevel & Mean Noise Level (dB), for each time \& height \\
\hline NumCodeBits & Number of Coded Bits for each mode \\
\hline NumCoherentIntegrations & Number of Coherent Integrations done for each mode \\
\hline NumFFt & Number of FFT points used for each mode \\
\hline NumSpectralAverages & Number of Spectral Averages done for each mode \\
\hline NyquistVelocity & Nyquist Velocity (m/s), for each mode \\
\hline Power & Uncalibrated Power (dB), for each time \& height \\
\hline PulseWidth & Pulse Width (ns), for each mode \\
\hline RadarConstant & Radar Constant (dB), hourly for each mode \\
\hline RangeCorrectedPowwer & Range-Corrected Calibrated Power (dBm), for each time \& height \\
\hline RxGain & Receiver Gain (dB) for each mode \\
\hline SignalToNoiseRatio & Signal-to-Noise Ratio (dB), for each time \& height \\
\hline SkyNoiseLevel & Sky Noise Level (dB), for each mode \\
\hline StartGateDelay & Delay to first range gate (ns) \\
\hline
\end{tabular}

See MMCR Data Stream Format Changes for differences between the new mmcrmom data stream fields and the mmcrcal, mmcrmoments fields. 


\subsubsection{Diagnostic Variables}

Table 5. Diagnostic Variables

mmcrmom Data Stream

\begin{tabular}{|l|l|}
\hline \multicolumn{1}{|c|}{ Variable } & \multicolumn{1}{c|}{ Description } \\
\hline AvgNoiseLevel & Average Noise Level (S/N <0), dB for each time \\
\hline CalCheckLevel & Receiver Calibration Check Level (dB) for each mode \\
\hline CalCheckTime & Time stamp for receiver calibration check \\
\hline ClutterHeight & Maximum height of clutter removal (m) \\
\hline DCFilterONOFF & DC Filtering on-off status \\
\hline DataQualityStatus & Data Quality Status, for each time (see Section 5.1.4) \\
\hline ModeDescription & Description of each radar mode \\
\hline NumReceivers & Total number of receivers in use, for each mode \\
\hline PeakTransmittedPowerAvg & $\begin{array}{l}\text { Peak transmitted power, averaged over an hour (dBm), for } \\
\text { each hour }\end{array}$ \\
\hline ReceiverMode & $\begin{array}{l}\text { For each mode, Identifies what multiple receiver mode does: } \\
\text { 0= single polarization } \\
1=\text { dual polarization }\end{array}$ \\
\hline 2= dual antenna \\
3= added loss in receiver (precip mode)
\end{tabular}

See MMCR Data Stream Format Changes for differences between the new mmcrmom data stream fields and the mmcrcal, mmcrmoments fields.

\subsubsection{Data Quality Flags}

DataQualityStatus (mmcrmom data stream only): DataQualityStatus has a value for each time value. It gives information on whether Reflectivity and RangeCorrectedCalibratedPower exist for this time and if so, how they were calibrated.

DataQualityStatus:

$1=$ No values for Reflectivity and RangeCorrectedCalibratedPower

2 = Abbreviated calibration was applied: Calibrated Power $=\mathrm{f}($ RxGain $)$

4 = Using default radar constant; No recent entry for Peak Power

$8=$ TWT fault during this file; Possible lost data 
qc_time (mmcrmom data stream only): Contains the results of quality checks on sample time. This field has a value at each sample time. The qc_time values are calculated by comparing each sample time with the previous time. In the table below, Delta_time $=\mathrm{t}[\mathrm{n}]-\mathrm{t}[\mathrm{n}-1]$.

qc_time:

$1=$ Delta_time is within expected interval.

$2=$ Delta_time is zero: Duplicate sample times

4 = Delta_time is greater than expected.

$8=$ Delta_time is less than expected.

TWTStatusCode (mmcrmom data stream only): This field reports on traveling wave tube (TWT) status once per hour. A nine-digit code is reported, as follows:

TWTStatusCode digit \#, from left to right:

Digits 1-3 = Percentage of time the TWT had peak power within acceptable bounds (0-100\%)

Digit $4=$ \# TWT retries at 55 minutes past the hour (values from 0-5)

Digit 5 = \# TWT retries at 45 minutes past the hour (values from $0-5$ )

Digit $6=$ \# TWT retries at 35 minutes past the hour (values from $0-5$ )

Digit 7 = \# TWT retries at 25 minutes past the hour (values from $0-5$ )

Digit $8=$ \# TWT retries at 15 minutes past the hour (values from $0-5$ )

Digit $9=$ \# TWT retries at 05 minutes past the hour (values from $0-5$ )

For example, a code of 072030020 indicates that $72 \%$ of the time TWT power was acceptable, there were 3 retries at 45 minutes past the hour and 2 retries at 15 minutes past the hour. Note that after 5 retries the TWT would be shut down due to a fault.

\subsubsection{Dimension Variables}

Table 6. Dimension Variables

mmcrmom Data Stream

\begin{tabular}{|l|l|}
\hline \multicolumn{1}{|c|}{ Variable } & \multicolumn{1}{c|}{ Description } \\
\hline alt & $\begin{array}{l}\text { Altitude, meters above mean sea level, of ground } \\
\text { instrument is sited on }\end{array}$ \\
\hline base_time & $\begin{array}{l}\text { Base time for file, in seconds since 1/1/1970 } \\
00: 00: 00 \text { GMT }\end{array}$ \\
\hline heights & $\begin{array}{l}\text { Range heights in meters above mean sea level of } \\
\text { data collection for each mode (center of radar } \\
\text { sample volume) }\end{array}$ \\
\hline lat & North latitude in degrees \\
\hline lon & East longitude in degrees \\
\hline time & $\begin{array}{l}\text { Time offset in seconds from midnight on file's } \\
\text { collection date }\end{array}$ \\
\hline time_offset & Time offset in seconds from base_time \\
\hline
\end{tabular}




\subsection{Annotated Examples}

Below are plots of Reflectivity, Mean Doppler Velocity, and Spectral Width, from top to bottom, for an SGP day. Areas of non-hydrometeor "clutter" are shown (typically insects, bits of vegetation or dust). Clutter is often seen at low levels at the SGP site and can be difficult to distinguish from hydrometeor echoes. The ARSCL VAP generally does a good job of identifying and eliminating clutter echoes (see Section 6.4 below for more information). The "bright band" is also indicated on the plot. This is an area of enhanced reflectivity caused by melting ice particles.

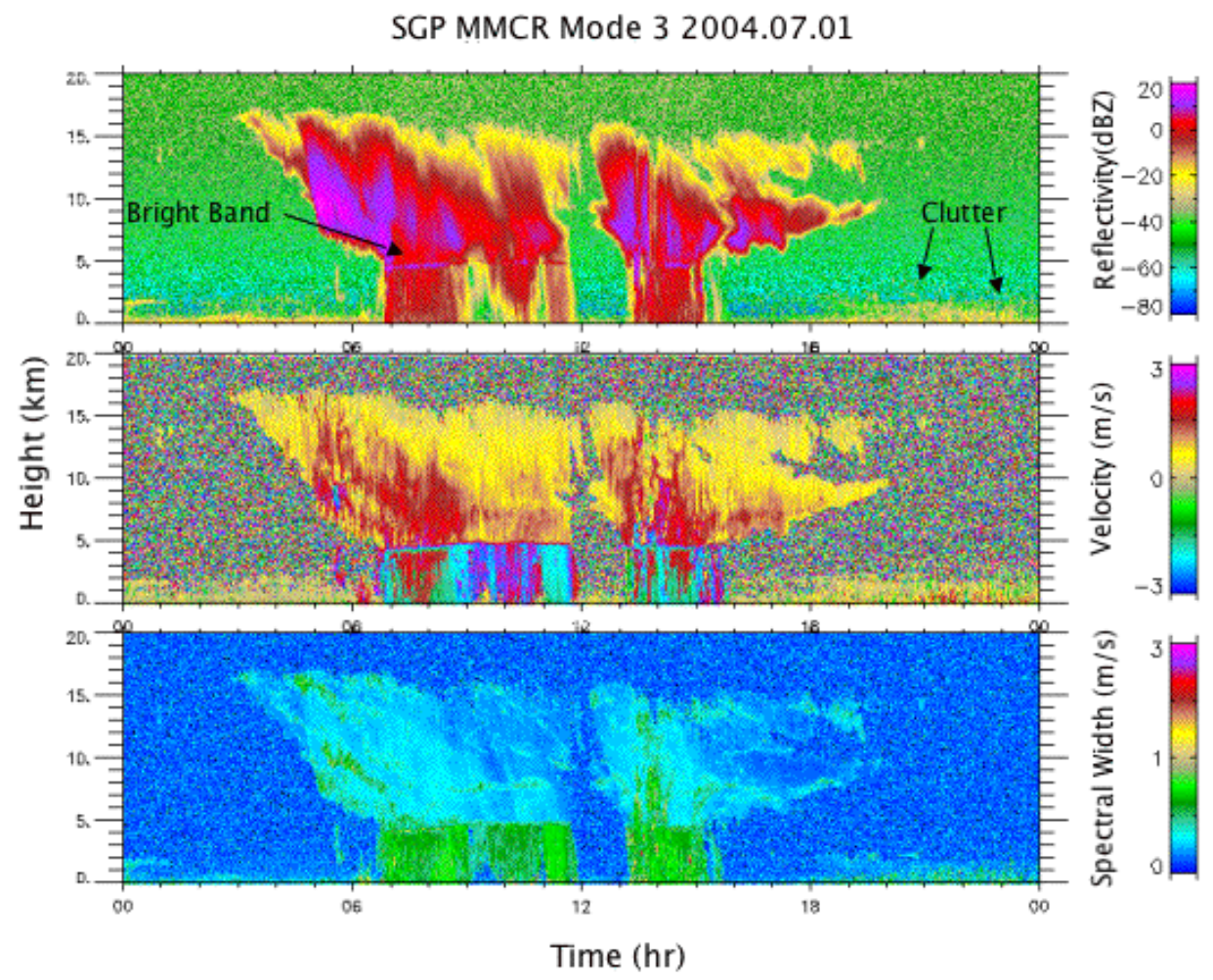

Figure 1.

Below are time vs. height plots of Reflectivity (for SGP 2004.02.29) for radar modes 1 (Boundary Layer), 2 (Cirrus), 3 (General) and 4 (Precipitation). Mode 1 samples the lowest kilometers only, but is more sensitive there than the other modes. Mode 2 is the most sensitive mode above 3 kilometers but sometimes has pulsecoding artifacts. These spiked artifacts in the presence of strong reflectivity gradients are caused by imperfect pulse decoding and can contaminate adjacent areas with weaker returns. Mode 3 is a good general mode, but is not quite as sensitive to thin clouds as Mode 2. Mode 4 is less sensitive than modes 2 and 3, but it does not saturate as readily in higher reflectivity regions, such as drizzle. 


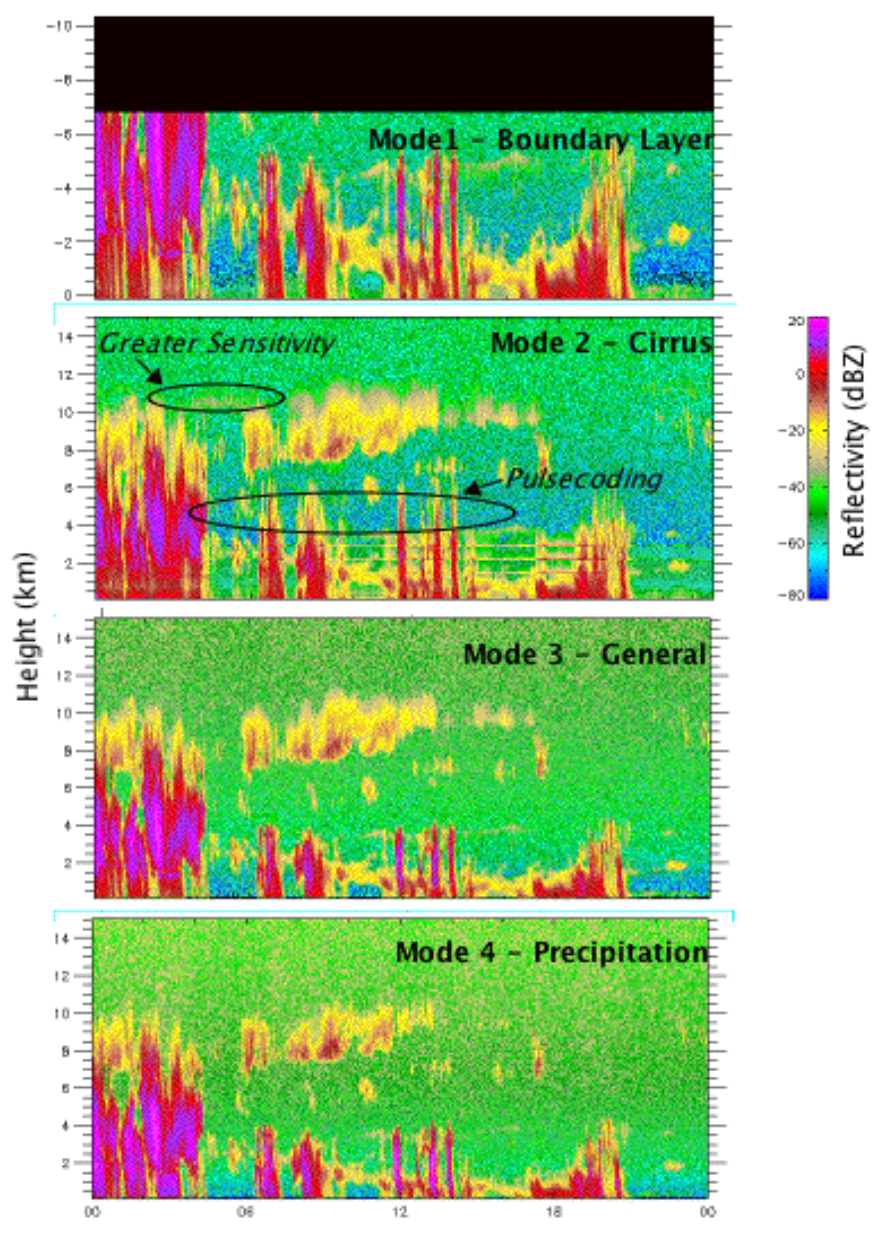

Figure 2. 


\subsection{User Notes and Known Problems}

MMCR processor upgrades are underway at all ARM Climate Research Facility (ACRF) sites. When the upgrade is done at a site, the MMCR data streams change from 'mmcrcal' and 'mmcrmoments' to a single data stream, 'mmcrmom.' The SGP and NSA sites were upgraded on 2003.09.09 and 2004.04.13, respectively. It is expected that the TWP sites will be upgraded in early 2005.

A comparison of the fields in the mmcrmom data streams with those in the mmcrcal and mmcrmoments data streams is available here:

http://www.gim.bnl.gov/armclouds/mmcr/mmcrMomNewVsOldDataStreams.html

MMCR spectral data, in data stream "mmcrspecmom” are also available at sites with the upgraded MMCR processor (currently SGP and NSA). The mmcrspecmom data can be ordered through the usual ARM Archive web "Power Interface.” But, please note that approximately 8 GB of spectral data are collected each day. Data requests covering a few hours to a few days will be accepted via the web interface. Spectral data availability at the archive lags several weeks behind data collection time because the data are periodically shipped to the archive on disks.

\subsection{Frequently Asked Questions}

\section{How is the minimum measurable height determined?}

There are two system delays that must be considered: the interval between the time when the pulse is transmitted and when the return signals are first sampled by the receiver detector (StartGateDelay variable in the moments and spectra files), and the time it takes the received signal to reach the detector from the receive antenna (RxDelay variable in the moments and spectra files). Hence, subtracting the RxDelay from the StartGateDelay yields the time that the pulse was moving through the atmosphere (transit time). The measurement height is $0.5 *$ transit_time. Thus,

Min. Meas. Hgt $=(0.5($ StartGateDelay - RxDelay $)) \times$ c

where the delays are expressed in seconds and $\mathrm{c}$ is the speed of light. For mode 1, StartGateDelay $=$ $1200 \mathrm{~ns}$ and RxDelay $=500 \mathrm{~ns}$, so 350e-9(3.0e8) = $105 \mathrm{~m}$ AGL (above ground level). In the data files, the heights are in MSL (mean sea level). The radar is located at approximately $318 \mathrm{MSL}$, so the lowest measurement height in the data is $105 \mathrm{~m}+318 \mathrm{~m}=423 \mathrm{~m}$.

\section{How does pulse coding affect the lowest measurement height?}

Essentially, complementary code renders the first $\mathrm{n}-1$ heights useless, where $\mathrm{n}$ is the number of coded bits. Therefore, an 8-bit code applied in a situation where the enhanced vertical resolution is $45 \mathrm{~m}$ means that the first usable data is from $8 * 45=360 \mathrm{~m}+105 \mathrm{~m}=465 \mathrm{~m}$ AGL. Note that $105 \mathrm{~m}$ is the height of the lowest measurement height. 


\section{Is the MMCR polarization diverse?}

Until the recent SGP MMCR upgrade, the MMCR has not been polarization-diverse at any sites. At the NSA and TWP sites, and at the SGP site prior to 2004.08.11, the MMCR transmits and receives at the same polarization; this is not adjustable.

As of 2004.08.11, the SGP is running a polarization mode, with polarization alternating from horizontal to vertical from pulse to pulse.

\section{What is the lifetime of the transmitter tube?}

The advertised lifetime for the Traveling Wave Tube Amplifier (TWTA) is 20,000 hours. This amounts to 2.3 years of continuous operation.

What index of refraction for water is used to computer reflectivity $(\mathrm{Kw})$ ?

\section{Data Quality}

\subsection{Data Quality Health and Status}

The Data Quality Office (DQO) website has links to several tools for inspecting and assessing MMCR data quality:

- DQ HandS (Data Quality Health and Status)

- DQ HandS Plot Browser

- $\underline{\text { NCVweb: }}$ Interactive web-based tool for viewing ARM data

Plots of reflectivity, Doppler radial velocity, and Doppler spectral width provide a good indicator of whether the system is operational or not.

\subsection{Data Reviews by Instrument Mentor}

Data reviews are done weekly. Monthly assessments will be provided here in the future.

\subsection{Data Assessments by Site Scientist/Data Quality Office}

All DQO and most Site Scientist techniques for checking have been incorporated within DQ HandS and can be viewed there.

\subsection{Value-Added Products and Quality Measurement Experiments}

Many of the scientific needs of the ARM Program are met through the analysis and processing of existing data products into "value-added” products or VAPs. Despite extensive instrumentation deployed at the ARM sites, there will always be quantities of interest that are either impractical 
or impossible to measure directly or routinely. Physical models using ARM instrument data as inputs are implemented as VAPs and can help fill some of the unmet measurement needs of the program. Conversely, ARM produces some VAPs not to fill unmet measurement needs, but to improve the quality of existing measurements. In addition, when more than one measurement is available, ARM also produces “best estimate” VAPs. A special class of VAP, called a Quality Measurement Experiment (QME), does not output geophysical parameters of scientific interest. Rather, a QME adds value to the input datastreams by providing for continuous assessment of the quality of the input data based on internal consistency checks, comparisons between independent similar measurements, or comparisons between measurement with modeled results, etc. For more information, see VAPs and QMEs.

The major MMCR-related VAP is called ARSCL, which stands for "Active Remote Sensing Cloud Layer.” ARSCL combines data from the MMCR, the micropulse light detection and ranging (MPL LIDAR), Vaisala ceilometer (VCEIL) and surface precipitation measurements to provide a time series of hydrometeor height distributions and best estimates of radar reflectivities, vertical velocities, and Doppler spectral widths. See the ARSCL VAP documentation and the ARM Tech. Memo for additional information.

\section{Instrument Details}

\subsection{Detailed Description}

\subsubsection{List of Components}

At all sites:

- $\quad$ Applied Systems Engineering TWTA

- $\quad$ Spacek Labs Coherent Up/Down Converter (CUDC)

- $\quad$ EMS Switching Circulators

- Alpha Cassegrain antenna

- 4-Port Circulators

- NOAA/ETL Pulse Controller

- Radian Receiver/Modulator

- Radian Interface

- IOtech Analog Multiplexer

- IOtech Analog-to-Digital Converter

- Tektronix Oscilloscope

NSA and SGP only:

- Windows NT Data Management System Computer, running LabView

- Windows NT Radar Control computer, running LapXM software 
TWP sites only:

- Solaris-PC Data Management System computer

- OS/2 Radar Control computer

\subsubsection{System Configuration and Measurement Methods}

The MMCR system consists of the radar, data acquisition/control subsystem, enclosures, cables, and accessories so that it will be operable in a semi-autonomous mode. For purposes of this specification, semi-autonomous operation is defined as a mode wherein an operator is required only to power up and power down the system. Once powered up, the MMCR will automatically enter a standby mode ready to begin taking data.

\subsubsection{Specifications}

\section{Radar specifications are as follows (Moran et al. 1997):}

Frequency

Peak Transmitted Power

Maximum Duty Cycle

Antenna Diameter

Antenna Gain

Beam Width (full-width, half-maximum)

PRF (max)
34.86 GHz (Wavelength 8.66mm, Ka-band)

$100 \mathrm{~W}$

$25 \%$

see table under Calibration History

see table under Calibration History

see table under Calibration History

$20 \mathrm{kHz}$

\section{MMCR Mode Sequence and Characteristics}

MMCR upgrades are under way at all sites, so details of mode characteristics and sequencing are changing. For detailed information on operational parameters at each site for specific time ranges, please see MMCR Operational Parameters.

Here the historical modes and sequencing will be discussed, followed by a description of recent changes at SGP and NSA.

\section{Current TWP and Historical NSA, SGP Mode Sequence}

Historically, the MMCR has cycled through 4 modes with combined dwell and processing times of approximately 9 seconds for each mode. The mode parameters have varied over time and at each site. For detailed information on operational parameters at each site for specific time ranges, please see MMCR Operational Parameters. As an example, here are the parameters in effect at SGP from September 1997 through August 10, 2004: 
Table 7. ARM SGP Site Operation Parameters 1997.09.15 - 2004.08.10

\begin{tabular}{|l|c|c|c|c|}
\hline \multirow{2}{*}{\multicolumn{1}{c|}{ Radar Parameter }} & \multicolumn{3}{c|}{ Operating Mode } \\
\cline { 2 - 5 } & $\begin{array}{c}\mathbf{1} \\
\text { Stratus }\end{array}$ & $\begin{array}{c}\mathbf{2} \\
\text { Cirrus }\end{array}$ & $\begin{array}{c}\mathbf{3} \\
\text { General }\end{array}$ & $\begin{array}{c}\mathbf{4} \\
\text { Precipitation }\end{array}$ \\
\hline Inter-Pulse Period (microsec) & 68 & 126 & 106 & 106 \\
\hline Pulse Width (microsec) & 0.3 & 0.6 & 0.6 & 0.6 \\
\hline Gate Spacing (microsec) & 0.3 & 0.6 & 0.6 & 0.6 \\
\hline Number of Gates & 110 & 167 & 167 & 167 \\
\hline Coherent Averages & 10 & 6 & 6 & 1 \\
\hline Spectral Averages & 64 & 21 & 60 & 29 \\
\hline FFT Length & 64 & 64 & 64 & 128 \\
\hline Coded Bits & 8 & 32 & 0 & 0 \\
\hline Dwell Time (sec) & 0.7 & 0.6 & 1.0 & 0.7 \\
\hline Obsv./Processing Time (sec) & 9 & 8.7 & 8.5 & 9 \\
\hline Minimum Detectable Signal (dBm) & $\sim-132$ & $\sim-132$ & $\sim-132$ & $\sim-132$ \\
\hline
\end{tabular}

\section{Current NSA Mode Sequence}

At NSA, the installation of the new C40 processor on 2004.04.14 was accompanied by a new mode sequence to provide more frequent boundary-layer (BL)sampling:

\section{BL GE BL CI BL GE BL PR}

Here BL is analogous to the Stratus mode, CI is the Cirrus mode, GE is the General mode, and PR is the Precipitation mode. The combined dwell and processing times for each mode are much shorter with the new processor, approximately 2 seconds per mode. The entire mode sequence completes in just under 18 seconds.

\section{Current SGP Mode Sequence}

At SGP, a dual-polarization mode was added on August 11, 2004. At this time the mode sequence and timing were also modified to provide more frequent BL sampling. A new precipitation mode was also added, with about $27 \mathrm{~dB}$ of additional loss in the receiver to reduce receiver saturation during light precipitation. The SGP mode sequence is:

\section{BL CI BL GE BL PR BL GE BL CI BL GE BL PO BL GE}

where BL is the boundary layer mode, $\mathrm{CI}$ is the cirrus mode, GE is the general mode, PR is the precipitation mode, and PO is the dual-polarization mode. Dwell and processing time for each mode is approximately 2 seconds, with the complete mode sequence completing in about 32.5 seconds. 


\subsection{Theory of Operation}

The MMCR works by transmitting a pulse of millimeter-wave energy from its transmitter through the antenna. The energy propogates through the atmosphere until it hits objects that reflect some of the energy back to the MMCR. These objects can be clouds, precipitation, insects, spider webs, man-made objects, etc. The same antenna is used to receive the return signal. The received signal is split into two channels, termed I and Q (for in-phase and quadrature). A digital signal processor processes these signals and provides power, Doppler velocity, and spectral width. The power measurement is processed by knowing the MMCR's calibration coefficient to provide the radar reflectivity.

Looking at the meteorlogical radar range equation gives insight as to how the MMCR works and what parameters affect its sensitivity. Any radar's sensitivity is proportional to the transmit power, the square of the antenna gain, and the square of the radar's wavelength. The sensitivity is inversely proportional to the square of the range from the radar to the target.

\subsection{Calibration}

\subsubsection{Theory}

There are several systems within the radar that require calibration at regular intervals. The values obtained from these calibrations are stored as constants, polynomials, or curves in the calibration files or programs. These are used by the software to convert raw radar moment files to range-corrected power $(\mathrm{dBm})$ and reflectivity (dBZ) data in netCDF format and sent to the site data system (SDS).

Below is a listing of the calibration constants stored in the system and the required frequency of calibration.

Table 8. Calibration Information

\begin{tabular}{|l|l|l|l|}
\hline \multicolumn{1}{|c|}{ Constant } & \multicolumn{1}{c|}{ Storage Location } & Interval (months) & \multicolumn{1}{c|}{ Storage Type } \\
\hline Inclinometer & MMCR Operating Computer & 120 & Polynomial \\
\hline RF noise diode & MMCR Data Computer & 12 & Constant \\
\hline Receiver Path Loss & Calibration Files & 12 & Constant \\
\hline Transmitter Path Loss & Calibration Files & 12 & Constant \\
\hline TWT RF Test Point & Manual & 60 & Constant \\
\hline Forward Power Monitor & MMCR Data Computer & 36 & Polynomial \\
\hline RF Attenuator & Internal & 36 & Polynomial \\
\hline IF Attenuator & Manual & 36 & Constant \\
\hline Radar Parameters & Calibration Files & 6 & Curve \\
\hline Noise Diode Path Loss & Manual & 36 & Constant \\
\hline Antenna Gain & Calibration Files & 60 & Constant \\
\hline Receiver Bandwidth & Calibration Files & 36 & Constant \\
\hline Range Delay & MMCR Operating Computer & 36 & Constant \\
\hline Coded Pulse Loss & Calibration Files & 36 & Constant \\
\hline
\end{tabular}




\subsubsection{Procedures}

This information is currently unavailable.

\subsubsection{History}

Antenna calibration information from the manufacturer:

Table 9. MMCR Antenna Table

\begin{tabular}{|c|c|c|l|c|c|}
\hline Diameter & Serial Number & Model \# & Location & Gain (dBi) & Beam Width \\
\hline $2 \mathrm{~m}$ & 11 & 63208400 & ARCS-1, Albuquerque & 53.37 & $0.32^{\circ}$ \\
\hline $2 \mathrm{~m}$ & 12 & 63208400 & SHEBA & 53.48 & $0.30^{\circ}$ \\
\hline $2 \mathrm{~m}$ & 13 & 63208400 & ARCS-2, Nauru & 52.73 & $0.31^{\circ}$ \\
\hline $2 \mathrm{~m}$ & 14 & 63208400 & Barrow, AK & 53.37 & $0.31^{\circ}$ \\
\hline $3 \mathrm{~m}$ & 11 & 63208300 & SGP & 57.48 & $0.19^{\circ}$ \\
\hline
\end{tabular}

\subsection{Operation and Maintenance}

\subsubsection{User Manual}

This information is currently unavailable.

\subsubsection{Routine and Corrective Maintenance Documentation}

SGP Preventative Maintenance Procedures can be accessed at this address: http://198.124.96.210/pm_proc/mmcrpm03.htm.

Preventive Maintenance Logs for SGP site instruments are available here:

http://198.124.96.210:591/cfdpm1/default.htm. Just click on the instrument desired then enter the date(s) of interest.

Corrective Maintenance Logs for SGP site instruments are available here:

http://198.124.96.210/menus/cmreports.html. Select the current fiscal year or historical period to access the maintenance database.

\subsubsection{Software Documentation}

Raw MMCR data are ingested at the Data Management Facility (DMF), creating netCDF a1 level data files, which are stored at the ARM Archive. Information on data file formats is available in Section 5, Data Description and Examples..

\subsubsection{Additional Documentation}




\subsection{Glossary}

See the ARM Glossary.

\subsection{Acronyms}

$\begin{array}{ll}\text { AAO } & \text { Adjacent Arctic Ocean } \\ \text { ACRF } & \text { ARM Climate Research Facility } \\ \text { AGL } & \text { above ground level } \\ \text { ARCS } & \text { Atmospheric Radiation and Cloud Station } \\ \text { ARM } & \text { Atmospheric Radiation Measurement (Program) } \\ \text { ARSCL } & \text { Active Remote Sensing Cloud Layer } \\ \text { CUDC } & \text { Coherent Up/Down Converter } \\ \text { DMF } & \text { Data Management Facility } \\ \text { DOE: } & \text { U.S. Department of Energy } \\ \text { DQO } & \text { Data Quality Office } \\ \text { ETL } & \text { Environment Technology Laboratory (NOAA) } \\ \text { IF } & \text { intermediate frequency } \\ \text { LIDAR } & \text { light detection and ranging } \\ \text { MMCR } & \text { millimeter cloud radar } \\ \text { MMW } & \text { millimeter wave (30GHz - 300GHz) } \\ \text { MPL } & \text { Micropulse Lidar } \\ \text { NOAA: } & \text { National Oceanic and Atmospheric Administration } \\ \text { NSA } & \text { North Slope of Alaska } \\ \text { PNNL } & \text { Pacific Northwest National Laboratory (Battelle) } \\ \text { QME } & \text { Quality Measurement Experiment } \\ \text { RF } & \text { radio frequency } \\ \text { SDS } & \text { site data system } \\ \text { SGP } & \text { Southern Great Plains } \\ \text { SHEBA } & \text { Surface Heat Budget of the Arctic Ocean } \\ \text { TWP } & \text { Tropical Western Pacific } \\ \text { TWTA } & \text { Traveling Wave Tube Amplifier } \\ \text { VAP } & \text { value-added product }\end{array}$

Also see ARM Acronyms and Abbreviations.

\subsection{Citable References}

Albrecht, B.A., TP Ackerman, G. Mace, D.W. Thomson, M.A. Miller, and R.M. Peters. 1991. “A surface-based cloud observing system.” Preprint Vol., Seventh Conf. on Meteorological Observations and Instrumentation, pp. 443-446, New Orleans.

Baum, B.A., T. Uttal, M .Poellot, T.P. Ackerman, J.M. Alvarez, J. Intrieri, D O’C. Starr, J Titlow, V. Tovinkere, and E.E. Clothiaux. 1995. "Satellite remote sensing of multiple cloud layers.” J. Atmos. Sci, 52:4210-4230. 
Bogush, A. 1989. Radar and the Atmosphere, Artech House.

Clothiaux, E.E., M.A. Miller, B.A. Albrecht, T.A. Ackerman, J. Verlinde, D.M. Babb, R.M. Peters, and W.J. Syrett. 1995. "An evaluation of a 94-GHz radar for remote sensing of cloud properties." J. Atmos.Ocean.Tech. 12:201-229.

Currie, N and C. Brown. 1987. Principles and Applications of Millimeter-Wave Radar, Artech House.

Dong X, TP Ackerman, E.E. Clothiaux, P. Pilewskie, and Y. Han. 1997. "Microphysical and radiative properties of boundary layer stratiform clouds deduced from ground-based measurements.” Journal Geophys. Res. 102:23829-23843.

Doviak, R.J., and D.S. Zrni. 1993. “Doppler Radar and Weather Observations.” 2 ed., Academic Press, p. 562.

Frish, A.S., C.W. Fairall, and J.B. Snider. 1995. "Measurement of stratus cloud and drizzle parameters in ASTEX with a Ka-band doppler radar and microwave radiometer.” J. Atmos. Sci. 52:2788-2799.

Hobbs, P.V., N.T. Funk, R.R. Weiss, J.D. Locatelli, and KR Biswas. 1985. "Evaluation of a 35- GHz radar for cloud physics research.” J. Atmos. Oceanic Technol. 2:35-48.

Intrieri, J.M., G.L. Stephens, W. Eberhard, and T Uttal. 1993. “A method for determining cirrus cloud particle sizes using a lidar and radar backscatter technique.” J. Appl. Meteor. 32:1074-1082.

Intrieri, J.M., WL. Eberhard, T. Uttal, J.A. Shaw, J.B. Snider, Y. Han, B.W. Orr, and S.Y. Matrosov. 1995. "Multiwavelength observations of a developing cloud system: the FIRE II 26 November 1991 case study.” J. Atmos. Sci. 52:4079-4094.

Kropfli, RA and RD Kelly. 1996. "Meteorological research applications of a mm-wave radar.” Meteorol. Atmos. Phys. 59:105-121.

Lhermitte, RM 1987. “A 94-GHz doppler radar for cloud observations.” J. Atmos. Oceanic. Tech. 4:36-48.

Lhermitte, R.M. 1987. "Small cumuli observed with a 3-mm wavelength doppler radar.” Geo. Research Letters 14:707-710.

Lhermitte, R.M. 1988. “Cloud and precipitation remote sensing at 94 GHz.” IEEE. Trans. Geo. Rem. Sen. 26:207-218.

Lhermitte, R.M. 1990. “Attenuation and scattering of millimeter wavelength radiation by clouds and precipitation.” J. Atmos. Oceanic. Tech. 7:464-479.

Liebe, H.J. 1985. “An updated model for millimeter wave propagation in moist air.” Radio Sci. 20(5):1069-1089. 
Liebe, H.J., T. Manabe, and G.A. Hufford. 1989. "Millimeter-wave attenuation and delay rates due to fog/cloud conditions.” IEEE Trans. Antennas Propagation 37(12):1617-1623.

Liebe, H.J. 1989. “MPM-An atmospheric milimeter-wave propagation model.” Int. J. Infrared and Millimeter Wave, 10(6):631-650.

Mace, G.G., D O'C. Starr, T.P. Ackerman, and P. Minnis. 1995. "Examination of coupling between an upper-tropospheric cloud system and synoptic-scale dynamics diagnosed from wind profiler and radiosonde data,” Journal of Atmospheric Sciences 52:4094-4127.

Martner, B.E. and R.A. Kropfli. 1993. "Observations of multi-layered clouds using Ka-band radar.” Proc. 31st Aerospace Sci. Mtg., AIAA, p. 8, Washington, D.C.

Matrosov, S.Y., T. Uttal, J.B. Snider, and R.A. Kropfli. 1992. "Estimation of ice cloud parameters from ground-based infrared radiometer and radar measurements.” J. Geophys. Res. 97:11567-11574.

Matrosov, S.Y. 1993. "Possibilities of cirrus particle sizing from dual-frequency radar measurements." J. Geophys. Res., 98, 20675-20683.

Matrosov, S.Y., AJ Heymsfield, JM Intrieri, BW Orr, and JB Snider. 1995. “Ground-based remote sensing of cloud particle sizes during the 26 November 1991 FIRE II cirrus case: comparisons with in situ data.” J. Atmos. Sci. 52:4128-4142.

Mead, J.B., R.E. McIntosh, D. Vandemark, and C.T. Swift. 1989. "Remote sensing of clouds and fog with a 1.4-mm radar.” J. Atmos. Oceanic Technol. 6:1090-1097.

Miller, M.A., M.P. Jensen, and E.E. Clothiaux. 1998. "Diurnal variability in the statocumulus transition region: a case study using 94 GHz radar.” J. Atmos. Sci.. 55:2294-2310.

Miller, M.A. and B.A. Albrecht. 1995. "Surface-based observations of cumulus-stratocumulus interaction during ASTEX.” J. Atmos. Sci. 52:2809-2826.

Moran, K.P., B.E. Martner, DC Welsh, DA Merritt, MJ Post, and T Uttal. 1997. “ARM’s cloud-profiling radar.: Proceedings of the 28th Conf. on Radar Meteorology, Austin, TX.

Pasqualucci F, BW Bartrum, RA Kropfli, and WR Moninger. 1983. :A millimeter-wavelength dualpolarization doppler radar for cloud and precipitation studies.: J. Climate Appl. Metero. 22:758-765.

Peters RM, BA Albrecht, MA Miller, and JT Treaster. 1992. “Automated cloud profiling with a $94 \mathrm{GHz}$ radar.” Proceedings of the Eleventh International Conference on Clouds and Precipitation, Montreal, Quebec, Canada. August 17-21, 1992.

Planck, V.G., D. Atlas, and W.H. Paulsen. 1955. :The nature and detectability of clouds and precipitation as determined by a 1.25 centimeter radar.” J. Meteor. 12:358-378. 
Post, M.J., K.P. Moran, and B Martner. 1996. Contractors for the Department of Energy ARM Program Millimeter-Wave Radars. Environmental Technology Laboratory, ERL, NOAA.

Probert-Jones, J.R. 1962. “The radar equation in meteorology.” Quart. J. Roy. Met. Soc. 88:485- 495.

Saugageot, H. 1992. Radar Meteorology, Artech House.

Sekelsky, S.M., and R.E. McIntosh. 1996. “Cloud Observations with a Polarimetric $33 \mathrm{GHz}$ and $95 \mathrm{GHz}$ Radar.” Meteorol. Atmos. Phys. 59:123-140.

Skolnik, M. 1990. Radar Handbook, Merril Skolnik, McGraw-Hill.

Uttal, T., J.M. Intrieri, W.L. Eberhard, T.P. Ackerman, and E.E. Clothiaux. 1995. "Cloud boundaries during FIRE II.” J. Atmos. Sci. 52:4276-4284.

Wexler, R. and D. Atlas. 1959. “Precipitation generating cells.” J. Meteor., 16:327-332. 